Favoriser les liens cours-stages

\title{
Défis et savoirs émergeant de discussions entre stagiaires et acteurs des milieux scolaire et universitaire dans le cadre d'un dispositif de coformation intermodulaire en enseignement
}

Auteures

Nadia, Cody, Université du Québec à Chicoutimi, nadia cody@uqac.ca

Sandra Coulombe, Université du Québec à Chicoutimi, sandra coulombe@uqac.ca

Andréanne Gagné, Université de Sherbrooke, andreanne.gagne4@sherbrooke.ca

Manon Doucet, Université du Québec à Chicoutimi, manon doucet@uqac.ca

Sophie Nadeau-Tremblay, Université du Québec à Chicoutimi sophie.nadeaut@csjonquiere.qc.ca

Collaborateurs

Suzie Boudreault, Annie De Champlain, Roxanne Labrecque, Andréa Lavoie, Caroline Leroux, Nadia Rose, Sonia St-Gelais, Isabelle Tremblay, Jérôme Tremblay ${ }^{1}$

\footnotetext{
${ }^{1}$ Nous ne pouvons passer sous silence la contribution, à notre projet de recherche, de Jérôme Tremblay, qui est décédé en février 2018. II était conseiller pédagogique à la Commission scolaire des Rives-du-Saguenay et était un membre important de notre équipe. C'était un homme rassembleur, dynamique et son professionnalisme faisait honneur à son travail. II nous manquera énormément.
} 


\section{REVUE HYBRIDE DE L'ÉDUCATION}

\section{Résumé}

La formation à l'enseignement privilégie plusieurs contextes d'apprentissage. Parmi ceux-ci, les stages constituent un terreau fertile pour développer les savoirs professionnels (Tardif et Lessard, 1999), où théorie et pratique s'enchevêtrent afin de former les futurs enseignants (Perrenoud, 2001). Dans les pratiques usuelles, les stagiaires vivent des situations dans lesquelles le partage du vécu se réalise entre étudiants d'un même module. Dans cette perspective, la volonté des étudiants a été exprimée à l'effet d'élargir leur réflexion avec d'autres stagiaires de différents modules d'enseignement. Les résultats de l'étude présentent des défis et des savoirs partagés dans le cadre d'un dispositif de coformation.

Mots-clés: savoirs professionnels; stagiaires en enseignement; collaboration université-milieu scolaire; codéveloppement professionnel; recherche-action-formation 


\section{$\&$ \\ REVUE HYBRIDE DE L'ÉDUCATION}

\section{Contexte}

Les besoins exprimés par des stagiaires en enseignement de l'Université du Québec à Chicoutimi (UQAC) à propos d'un lieu et d'un temps pour échanger entre eux, autour de situations vécues en stage, constituent le point de départ de cette recherche participative. La requête formulée par des stagiaires du Baccalauréat en éducation préscolaire et en enseignement primaire (BEPEP) portait sur un besoin d'échanges entre étudiants, dépassant les limites d'une cohorte ou d'un module, de façon intra, mais également intermodulaire, pour "conseiller et être conseillés " par leurs pairs, mais aussi par les acteurs clés qui les accompagnent. Des données émergentes des évaluations de trois modules en enseignement, soit le BEPEP, le Baccalauréat en enseignement en adaptation scolaire et sociale (BEASS) et le Baccalauréat en enseignement secondaire (BES), ont mis ce même besoin en évidence. Elles suggéraient également de prioriser la mise en place d'un mécanisme ou d'un dispositif pour un arrimage plus soutenu entre les cours et les stages, notamment en rapprochant les milieux universitaire et scolaire, et ce, afin de faire face aux défis de l'insertion professionnelle.

\section{Problématique}

Au Québec, des chercheurs tels que Coulombe, Zourhlal et Allaire (2010), Mukamurera, Martineau, Bouthiette et Ndoreraho (2013) ainsi que Rojo et Minier (2015) ont documenté les défis rencontrés par les nouveaux enseignants en processus d'insertion professionnelle. Ces défis portent sur l'acte d'enseigner, la gestion de classe, les élèves ayant des besoins particuliers et les rôles et responsabilités liés à la tâche enseignante (Coulombe et al., 2010). Rojo et Minier (2015) notent, en plus de ceux relevés, des défis liés à la diversification disciplinaire des tâches professionnelles et aux structures organisationnelles et institutionnelles, ainsi qu'à la quête de performance. Pour leur part, Mukamurera et al. (2013) recensent des programmes d'insertion des enseignants qui correspondent aux besoins et aux difficultés rencontrés par les nouveaux enseignants. Ces difficultés rejoignent celles recensées, en plus de mettre l'accent sur la détresse psychologique relative à la tâche et au manque d'expérience, de même que sur les conditions difficiles d'insertion.

II semble que les défis soient les mêmes dans d'autres systèmes d'éducation. De Stercke et al. (2010) les résument en trois catégories de difficultés, soit les difficultés administratives, organisationnelles et matérielles, les difficultés pédagogiques et didactiques et les difficultés relationnelles. Duchesne et Kane (2010) retiennent des défis générés par la gestion de classe et par la gestion de l'enseignement. Vanhulle (2012) nuance ce constat en affirmant que les nouveaux enseignants prioriseraient surtout les premiers, ainsi que ceux d'ordre relationnel, plutôt que les seconds. Ces défis seraient toutefois plus faciles à surmonter 


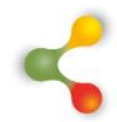

\section{REVUE HYBRIDE DE L'ÉDUCATION}

lorsque les enseignants peuvent recourir au soutien de leur mentor ou des autres enseignants de l'école (Duchesne et Kane, 2010).

Considérant les défis de l'insertion professionnelle, des questions se posent vis-à-vis du rôle de la formation initiale à l'enseignement. Même avec des stages en milieu de pratique, répartis sur quatre années de formation, il semble que cette dernière ne parvient pas à préparer pleinement les étudiants à la réalité de l'enseignement. Dans ces circonstances, Desbiens, Spallanzani et Borges (2012) interrogent le réalisme des attentes vis-à-vis des stagiaires. En effet, ceux-ci doivent développer les compétences professionnelles attendues de l'enseignant, ce qui passe par l'établissement de relations significatives avec les élèves et l'enseignant associé, le pilotage de situations d'enseignementapprentissage, le recadrage des perceptions idéalistes, la construction de leur identité, leurs valeurs, leurs savoirs, leurs aspirations, et plus encore. Cody (2013), Malo (1997), Morales Perleza (2016) et Perrenoud (2001) montrent que le développement de compétences professionnelles doit résulter d'une interaction constante entre la théorie et la pratique. Or, lorsque vient le temps de réaliser ces liens, le stagiaire éprouve des difficultés, car il accorde plus d'importance aux savoirs expérientiels qu'aux savoirs formels (Martin, 2002). Perez-Roux (2016) soutient également que le développement des compétences professionnelles engage le sujet dans un processus d'appropriation de savoirs pluriels et composites. Selon Balslev (2016), les défis et les préoccupations des jeunes enseignants restent constants entre la dernière année de formation initiale et la première année en emploi ; ce sont les savoirs mobilisés pour y faire face qui varient. L'auteur relève des savoirs de référence académique et des savoirs issus de stages pendant la formation, puis des savoirs institutionnels provenant de l'expérience et des collègues lors de la première année en emploi. On peut en déduire que l'enseignant novice convoque peu les savoirs académiques si le contexte ne l'y contraint pas et qu'il ne les perçoit peutêtre pas comme utiles lors de ses premières années de formation.

Dans cette perspective, la priorité irait donc aux savoirs issus de la pratique plutôt qu'à ceux issus de la recherche (Perrenoud, Altet et Lessard, 2008 ; Vanhulle, 2013). À cet effet, Balslev (2016) postule que d'ajouter des dispositifs complémentaires aux stages réalisés dans les milieux de pratique contribuerait à l'apprentissage professionnel à travers les commentaires, les questions et les réflexions des formateurs et des collègues.

Dans le cadre des programmes de formation à l'enseignement, différents dispositifs permettent d'accompagner les futurs enseignants de manière à ce que ceux-ci développent leurs compétences professionnelles. Une rapide recension des écrits permet de répertorier des dispositifs basés sur l'énonciation, dont l'argumentation pratique (Fenstermacher et Richardson, 1994), l'entretien de stage (Balslev, 2016), la coévaluation 


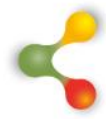

\section{REVUE HYBRIDE DE L'ÉDUCATION}

(Colognesi et Van Nieuwenhoven, 2017), ainsi que l'entretien d'explicitation (Vermersch, 1994) ou l'entretien d'autoconfrontation (Clot, Faïta, Fernandez et Scheller, 2001; Theureau, 2000). Coexistent avec cette première catégorie de dispositifs d'accompagnement ceux qui s'appuient sur l'activité scripturale. Nous référons alors aux portfolios papier ou numériques, aux journaux de bord, à l'écriture réflexive, aux forums de discussion et aux interactions en ligne (Collin, 2010). Enfin, une autre catégorie se distingue, celle des dispositifs collaboratifs qui mettent en relation plusieurs personnes. Les recherches menées par Coulombe, Allaire et Zourhlal (2016), Coulombe, Doucet, Zourhlal et Thibeault (2017) ainsi que Coulombe et al. (2010) suggèrent que des dispositifs collaboratifs, tels que des communautés de pratique virtuelle ou des groupes de codéveloppement professionnel, permettent d'accompagner les nouveaux enseignants, notamment parce qu'ils favorisent le partage de savoirs professionnels issus de l'expérience en enseignement.

Compte tenu des demandes effectuées par les étudiants du BEPEP, à savoir de discuter autour du vécu en milieu de stage, de façon intra, mais également intermodulaire, pour "conseiller et être conseillés" et de la nécessité de collaboration entre l'université et le milieu scolaire pour accompagner les étudiants dans ces discussions, nous posons les questions de recherche suivantes: Quels savoirs partagés par les stagiaires contribuent à développer une culture de collaboration? Quelles stratégies collaboratives université-milieu scolaire permettent la diversification et la mobilisation des savoirs partagés par les stagiaires ? Le présent article présente les résultats de l'analyse des données relatives à la première question de recherche, laquelle a fait l'objet de la première année du projet.

\section{Cadre conceptuel}

La notion de savoirs professionnels définie dans cet article constitue le concept clé de l'étude. Nous considérons, d'abord, les savoirs professionnels comme des ressources que construit un individu au cours de son expérience (Breithaupt, 2015 ; Pastré, Mayen et Vergnaud, 2006). Les savoirs professionnels sont culturels, rationalisés et font l'objet de pratiques discursives (Tardif et Lessard, 1999). Morales Perleza (2016) cite notamment Verloop, Van Driel et Meijer (2001) pour définir la base de connaissances comme un ensemble de savoirs multiples, lesquels proviennent de différentes sources formelles et informelles. Les sources formelles renvoient à la formation universitaire, alors que les sources informelles se rapportent à l'expérience vécue. Malo (2005), en référence à la théorie de l'agir professionnel de Schön (1994), suggère que ces savoirs forment un répertoire pour l'action, comme un ensemble de référents contextuels à la pratique, structurés par ce contexte et susceptibles d'être restructurés par les nouvelles situations rencontrées. 


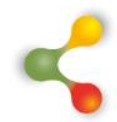

\section{REVUE HYBRIDE DE L'ÉDUCATION}

Plusieurs auteurs proposent différentes catégories de savoirs. Par exemple, Vanhulle (2012) et Garnier (2008) parlent des savoirs "à " enseigner, comme de savoirs didactisés (savoirs disciplinaires) et des savoirs «pour » enseigner, comme ceux faisant l'objet d'une appropriation didactique (modalités pédagogiques). Perrenoud, Altet et Lessard (2008) relèvent également des savoirs issus de la recherche ou théoriques et des savoirs issus de la pratique ou de l'expérience ou professionnels. Plus explicitement, Breithaupt (2015), Vanhulle $(2009,2012)$ et Clerc (2013) retiennent quatre types de savoirs de références : les savoirs académiques qui regroupent l'ensemble des savoirs construits et diffusés par la recherche comme les théories, les concepts scientifiques, les principes pédagogiques ou les recherches référencées par la formation; les savoirs institutionnels qui couvrent les textes prescriptifs de la profession enseignante comme les lois ou les référentiels ; les savoirs de la pratique qui semblent reconnus par les praticiens ou faire consensus, souvent tacites et peu formalisés, ils se transmettent de l'expert au novice ; enfin, les savoirs expérientiels qui sont ceux issus de l'expérience propre de l'individu, qui font l'objet par l'explicitation d'une certaine formalisation.

À l'instar de Gagné (2019), Koehler et Mishra (2009), Mialaret et Barbier (2011), Morales Perleza (2016) et Vanhulle (2009), nous retenons, aux fins de notre recherche, dix types de savoirs professionnels propres aux enseignants. Nous définissons les savoirs théoriques (1) par des références académiques et scientifiques propres aux sciences de l'éducation ; les savoirs disciplinaires (2) par les savoirs liés au contenu des différentes disciplines (matières) à enseigner ; les savoirs curriculaires (3) par les savoirs proposés dans les programmes de formation; les savoirs didactiques (4) par les stratégies pour enseigner les contenus disciplinaires ; les savoirs pédagogiques (5) par les connaissances portant sur la gestion et l'organisation de la classe, les méthodes et les stratégies pédagogiques à mettre en place pour faire apprendre ; les savoirs sur les élèves (6) par la connaissance des caractéristiques, des besoins des élèves; les savoirs technologiques (7) par la connaissance de la technologie pour favoriser l'apprentissage et aider à l'enseignement; les savoirs contextuels (8) par les connaissances relatives au contexte de la classe, de l'école ou de la profession; les savoirs éducatifs (9) par les connaissances liées aux finalités de l'éducation, ainsi qu'aux objectifs, aux fondements et aux valeurs de l'école ; et les savoirs expérientiels (10) par ceux construits dans la pratique du métier, qu'ils soient implicites ou explicites, validés par la pratique, la théorie ou la réflexion sur les liens théorie-pratique.

Vanhulle (2012) rappelle toutefois que l'enseignant ne possède pas de tiroirs dont il extrait, en situation d'action et d'interaction, des savoirs propres à intégrer l'ensemble de ce qui doit être appris et le processus pour y parvenir. L'essentiel pour Vanhulle (2013) s'avère donc d'amener l'étudiant en formation à l'enseignement à faire des liens entre les cours et les stages, des liens entre les savoirs de référence acquis dans le contexte 


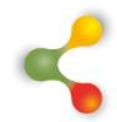

\section{REVUE HYBRIDE DE L'ÉDUCATION}

académique et les expériences menées dans le milieu de travail. De cette manière, il peut développer ses compétences professionnelles à l'intérieur de différents cadres sociaux, institutionnels et académiques. L'intégration des savoirs professionnels construits exige ainsi un travail réflexif. La mise en place d'un dispositif d'accompagnement collaboratif, basé notamment sur une discussion volontaire ou suscitée, apparait tout indiquée pour favoriser ces apprentissages.

Le deuxième concept défini dans le cadre de cette étude, soit la collaboration, sert, quant à lui, à mettre en perspective le problème de recherche. Nous considérons la collaboration, par son intensité, son mode de fonctionnement et ses formes de communication (Beaumont, Lavoie et Couture, 2010 ; Dionne et Savoie-Zajc, 2011 ; Marcel, Dupriez et Bagnoud, 2007). II s'agit d'un processus relationnel (Larivée, Kalubi et Terrisse, 2006), d'interactions plus ou moins intenses entre des individus autour d'un but commun (Corriveau et Savoie-Zajc, 2010) et d'un espace permettant d'amorcer un travail conjoint (Cody, Coulombe, Giroux, Gauthier et Gaudreault, 2016). La collaboration amène à la connaissance de soi comme professionnel engagé dans le dialogue et la communication avec les collègues qui partagent des objectifs communs (Bush, 2003, cité par Borges, Portelance et Pharand, 2011). Lessard, Kamanzi et Larochelle (2009) soulignent que les enseignants ont besoin d'être formés sur l'intérêt et la manière de collaborer entre eux pour améliorer leurs modes de transmission des savoirs, d'évaluation et de formation des élèves. Autrement dit, les habiletés nécessaires à la collaboration ne peuvent s'acquérir uniquement dans l'expérience; il faut l'apprendre systématiquement par la voie de la formation initiale et continue.

À la suite de la présentation des concepts principaux utilisés pour appréhender le problème à l'étude, nous énonçons les deux objectifs de recherche. Premièrement, la recherche vise à dégager et analyser les savoirs partagés par les stagiaires relativement à l'acte d'enseigner, à la gestion de la classe, aux élèves à besoins particuliers ainsi qu'au rôle respectif des intervenants en milieu scolaire (an 1). Par la suite, elle s'emploie à identifier des stratégies collaboratives permettant la diversification et la mobilisation des savoirs partagés répertoriés (an 2). Comme mentionné antérieurement, le présent article porte plus particulièrement sur les données recueillies lors de la première année du projet de recherche, c'est-à-dire sur les savoirs partagés par les stagiaires de différents modules relativement aux défis rencontrés dans le cadre de leur

stage.

\section{Méthodologie}

Pour atteindre ces objectifs, une recherche-action-formation a été privilégiée (Paillé, 1994 ; Prud'homme, Presseau et Dolbec 2007). L'étude répond aux exigences de ce type de recherche puisqu'elle vise à former 


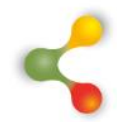

\section{REVUE HYBRIDE DE L'ÉDUCATION}

les participants en plus de collecter des données de recherche en vue de la production de connaissances (Paillé, 1994 ; Prud'homme, 2007).

Dans le cadre de la présente étude, le parcours de la rechercheaction-formation sous-tend la mise en œuvre d'un dispositif de coformation: le groupe de codéveloppement professionnel (Payette et Champagne, 1997) accompagné (L'Hostie, Cody et Laurin, 2011), lequel mise sur les interactions entre les participants afin d'améliorer leur pratique professionnelle. Ce processus d'échanges se structure autour de six étapes qui sont l'exposé de la problématique, la clarification de la problématique avec les questions d'information, l'établissement du contrat de consultation, les réactions, commentaires et suggestions des pairs (et des accompagnateurs), la synthèse et le plan d'action, l'identification des apprentissages avec l'évaluation de la rencontre. Ce déroulement permet de produire des connaissances relatives aux pratiques des stagiaires par l'analyse de situations professionnelles vécues en leur offrant l'occasion de se former, de se développer professionnellement en apprenant les uns des autres et de profiter de l'expertise d'accompagnateurs.

À travers l'analyse de situations vécues, le groupe a mobilisé différents types de savoirs afin de traiter les cas présentés. Le dispositif a agi, par son contexte, sur les aptitudes sociales comme l'entraide et la collaboration, pour favoriser les apprentissages chez les étudiants.

Au total, 36 stagiaires de toutes années de formation et provenant de trois modules en enseignement de l'UQAC (BEPEP, BEASS et BES) ont répondu positivement à notre invitation et 28 ont participé au dispositif. D'une durée d'environ 90 minutes, cinq groupes de codéveloppement professionnel accompagnés ont eu lieu à trois moments différents en 20172018, soit en décembre (avant la majorité des stages), en février (à la mistage) et en mars (un peu avant la fin des stages). Ces rencontres ont été réalisées en présence à I'UQAC. Trois chercheuses, responsables de la direction des modules concernés, ainsi que huit intervenants faisaient partie de l'équipe de recherche et provenaient des quatre commissions scolaires de la région Saguenay-Lac-Saint-Jean. Ainsi, trois chercheuses, quatre conseillers pédagogiques, une direction d'école et trois enseignantes, dont deux ayant déjà agi à titre de superviseures universitaires, ont animé et accompagné ces groupes composés d'une dizaine de stagiaires selon les six étapes de Payette et Champagne (1997). On parle donc ici d'une coresponsabilité de collaboration des milieux de formation.

Toutes les discussions ont été enregistrées sur support audio et le corpus de données a été transcrit en verbatim. Chaque intervention ou prise de parole par un participant du groupe a constitué un segment, pour un total de 1886 segments, dont 721 ont permis d'identifier des défis et des savoirs professionnels. De plus, des assistantes de recherche ont participé 


\section{6}

\section{REVUE HYBRIDE DE L'ÉDUCATION}

aux rencontres pour consigner, à partir des échanges en groupe, des notes d'observation servant à élaborer un premier canevas d'ensemble de chacune des six étapes du dispositif. Le but de la recherche n'étant pas de spécifier ou de comparer l'apport des stagiaires des différents programmes, cette variable n'a pas été prise en compte pendant la collecte. II n'y a pas eu lieu d'interroger l'apport des stagiaires par module, puisque c'est l'essence des propos partagés qui était appréhendée. De façon générale, l'expertise des uns a servi à enrichir la réflexion des autres.

La méthode de l'analyse qualitative par catégories conceptualisantes (Paillé et Mucchielli, 2008) a été utilisée par la suite pour traiter et structurer le matériel empirique des rencontres en groupe de codéveloppement. Chaque segment a été analysé afin de faire émerger les principaux défis évoqués par l'interlocuteur (768 codes attribués), ainsi que les savoirs partagés (836 codes attribués). Cette analyse a porté sur le contenu explicite du discours. Enfin, deux séances de covalidation ont été réalisées sur des extraits du corpus de données de manière à valider l'interprétation faite de ces dernières par l'ensemble des membres de l'équipe de recherche. La section suivante présente les résultats liés aux principaux défis et savoirs professionnels ayant émergé de chacun des cinq groupes de codéveloppement.

\section{Résultats}

Cet article expose les résultats d'une recherche-action-formation mettant en lumière la co-construction (Dionne et Savoie-Zajc, 2011) et le partage de savoirs professionnels issus de l'expérience et de la formation dans le cadre d'un dispositif de coformation qu'est le groupe de codéveloppement professionnel accompagné. Pour ce faire, nous exposons brièvement le cas discuté lors de chacun des cinq groupes de codéveloppement professionnel et tentons de faire ressortir les défis et les savoirs professionnels partagés lors de ces discussions. Les thématiques abordées lors des rencontres sont inspirées des situations professionnelles vécues par les stagiaires et sont liées à l'acte d'enseigner, à la gestion de classe, aux contextes social et scolaire et à la construction de l'identité professionnelle. De façon plus particulière, les sujets suivants ont été abordés : la désorganisation dans une classe, les attentes du superviseur universitaire versus les besoins des élèves, la douance, le trouble du déficit de l'attention avec hyperactivité (TDAH) et un trouble de comportement versus un système d'émulation complexe.

\section{Premier cas}

Le premier cas est lié à une difficulté relative à la gestion de classe. La situation se déroule au cours d'une évaluation du stagiaire alors qu'il réalise son deuxième stage avec un groupe d'élèves du secondaire. Ce 


\section{8}

\section{REVUE HYBRIDE DE L'ÉDUCATION}

groupe est considéré assez difficile. Alors que le superviseur universitaire se présente en classe pour observer le stagiaire, les élèves de la classe se désorganisent lors d'une transition entre deux activités autour de la $45^{\mathrm{e}}$ minute de cours. Devant la difficulté à reprendre le contrôle de la classe, le stagiaire opte pour arrêter toute activité et exige des élèves qu'ils restent en silence pendant les 15 dernières minutes du cours, et ce, malgré la situation d'évaluation dans laquelle il se trouve. Par rapport à cette situation, l'étudiant a invité les participants de son groupe de codéveloppement à lui proposer d'autres interventions possibles.

Lors des échanges concernant ce cas, nous avons relevé le défi de la gestion de classe 96 fois et celui de l'acte d'enseigner 47 fois. En ce qui a trait aux savoirs professionnels partagés, ils se rapportaient principalement à la pédagogie et à l'expérience. En fait, 62 codes se regroupent dans la catégorie des savoirs pédagogiques et 48 dans les savoirs expérientiels. Par exemple, un participant au groupe de codéveloppement professionnel énonçait une solution liée au défi de la gestion de classe en mobilisant un savoir pédagogique dans ces mots :

Au début, il faut établir des règles avec les élèves. Celles qu'ils voudraient dans la classe puisque c'est important de ne pas trop en mettre, mais qu'elles soient liées à une conséquence. Lorsque l'élève ne respecte pas les règles, il y a une conséquence qui vient avec cette règle. L'élève peut même la décider lui-même avec l'accord de l'enseignant bien sûr, mais elle doit être cohérente avec l'acte ou l'action posée (G1;E95).

Un autre participant énonçait son point de vue lié à l'acte d'enseigner en référant à des savoirs de type expérientiel de la manière suivante :

Lorsque je vois que les élèves, ça ne leur tente pas, je vais aller dans leur sens, mais je ne vais pas leur laisser le choix. C'est nous qui dirigeons la classe et nous devons toujours avoir un plan B comme des feuilles d'activités liées au module que tu enseignes. Avec un groupe qui ne veut rien faire, tu peux aussi leur offrir de continuer jusqu'à cinq minutes avant la cloche et après ça leur laisser cinq minutes pour jaser ensemble. Ils pensent qu'ils ont un choix, qu'ils peuvent décider et ils sont contents, mais dans le fond, c'est nous qui décidons (G1;E128).

\section{Deuxième cas}

Le deuxième cas met en lumière des difficultés à adopter la bonne posture par un stagiaire. Une stagiaire de troisième année en enseignement en adaptation scolaire réalise son stage dans une classe en 


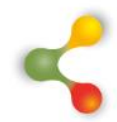

\section{REVUE HYBRIDE DE L'ÉDUCATION}

région éloignée. Selon sa perception, elle doit prendre en charge la classe et répondre aux besoins des élèves, ce que lui confirment le guide de stage et le superviseur universitaire. Au fil du stage, elle prend conscience qu'en travaillant toujours en collaboration avec son enseignante associée, elle peut répondre davantage aux multiples besoins des élèves. Cependant, les attentes du stage exigent qu'elle y parvienne seule. Elle se questionne donc sur la posture à adopter en tant que stagiaire. Doit-elle privilégier le service aux élèves ou répondre aux exigences inscrites dans le guide de stage?

L'analyse des données recueillies à propos de ce cas montre 44 codes attribués aux défis des rôles des intervenants dans le cadre des stages, 38 relatifs au contexte de formation et 37 liés à l'acte d'enseigner. Les savoirs collaboratifs (30) et identitaires (29) sont les principaux savoirs émergeant de l'analyse de ce cas. Un extrait relève notamment le défi lié aux rôles des intervenants et la place des savoirs collaboratifs :

En stage, le superviseur doit absolument évaluer à partir de quelque chose, c'est pour ça qu'il y a des critères. II comprend notre réalité, qu'on fait pas juste ce qui est demandé et que l'on en fait plus pour aider. Je trouve qu'il faut vraiment se parler, trouver des ententes entre la direction, les divers intervenants et les différents acteurs sociaux pour trouver des arrangements et savoir comment s'y prendre pour que tout le monde soit bien là-dedans. La meilleure chose, c'est la communication! (G2 : E117).

De la même manière, un autre participant énonçait les propos suivants, se rapportant davantage au contexte de formation et aux savoirs identitaires :

J'ai des valeurs et je ne savais pas vers quoi me diriger parce que je suis une personne perfectionniste qui aime bien faire les choses et qui veut être bonne pendant son stage. Donc, d'un côté je me dis que c'est ce que le superviseur veut, qu'il va m'évaluer et je comprends qu'il n'a pas le choix. D'un autre côté, ma valeur humaine, ma raison d'être en adaptation scolaire, c'est parce que je veux, du mieux possible, répondre aux besoins des élèves pis je ne suis pas capable de le faire en répondant aux exigences du stage (G2;E34).

\section{Troisième cas}

Le troisième cas présenté dans ce texte expose une situation rencontrée en stage relativement à une élève douée. Une étudiante au BEPEP a en effet partagé une situation où une fillette manifeste un haut 


\section{8}

\section{REVUE HYBRIDE DE L'ÉDUCATION}

potentiel pour son âge. Malgré plusieurs stratégies mises en place par les enseignantes, l'enfant semble se désintéresser de l'école. À la veille de réaliser son quatrième stage, l'étudiante se demande comment elle peut aider un élève doué alors que des enseignants expérimentés n'y arrivent qu'en partie.

L'analyse de ce cas a permis de recenser 53 codes relatifs aux défis liés aux élèves à besoins particuliers et 31 à celui de l'acte d'enseigner. 31 codes ont également été repérés concernant les savoirs sur les élèves et 29 à propos des savoirs pédagogiques. La citation suivante montre un défi lié aux élèves à besoins particuliers et un partage de savoirs sur les élèves :

Elle a des amis, mais elle ne comprend pas comment ils raisonnent. Cela lui nuit un peu puisque ses amis ne sont pas capables de la suivre, mais elle a une meilleure amie qu'elle voit tout le temps. Puis, ce n'est pas une enfant rejetée. Cela a plutôt des répercussions en ce qui a trait à son développement cognitif (G3 ;E22-23).

Un participant énonçait également des propos liés à l'acte d'enseigner et aux savoirs pédagogiques dans les mots suivants :

L'élève douée, oui elle termine avant, mais il y en a d'autres sans la douance qui vont terminer avant et qui ne le disent pas. On ne les voit pas. Avec mes élèves, ce que je fais souvent, c'est que j'ai une place en arrière de la classe pour le matériel. On prend connaissance des projets ensemble, le fonctionnement, le défi et l'évaluation. C'est à l'élève, avec l'enseignant, de choisir comment il va réaliser ce projet. Dans ces projets, ce que j'aime, c'est le choix de présentations. Ce n'est pas de faire un travail pour le travail, c'est de le faire en ayant un objectif au bout du compte et un apprentissage (G3 ;E55).

\section{Quatrième cas}

Le quatrième cas présente des difficultés rencontrées par une stagiaire relativement à des interventions auprès d'un élève ayant des besoins particuliers. La situation se déroule pendant un deuxième stage dans une classe ordinaire au secondaire. La stagiaire en adaptation scolaire s'insère dans une classe où le climat entre l'enseignant et les élèves semble difficile. Un jour, un élève ayant un trouble du déficit de l'attention avec hyperactivité (TDAH) dérange les autres élèves et malgré les avertissements continus de la part de la stagiaire, ses comportements perturbent le groupe-classe. La stagiaire l'envoie donc se calmer dans le corridor. Cette deuxième intervention ne fonctionne pas et l'élève est donc dirigé vers un local d'accueil. À son retour en classe, l'enseignant associé 


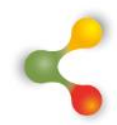

\section{REVUE HYBRIDE DE L'ÉDUCATION}

n'est pas satisfait de la gestion de la situation en précisant que l'élève n'avait simplement pas pris sa médication. La stagiaire se questionne : un élève TDAH est-il imputable de ses actes lorsqu'il ne prend pas sa médication?

Les éléments de la discussion qui ont suivi ce questionnement ont porté 58 fois sur les élèves à besoins particuliers et 44 fois sur les rôles des intervenants, de même que 39 codes ont été associés aux savoirs collaboratifs, 32 aux savoirs pédagogiques et 20 aux savoirs sur les élèves. Des propos extraits du verbatim illustrent la teneur des échanges relativement aux élèves à besoins particuliers et aux savoirs sur les élèves. C'est le cas d'un participant qui relatait :

Effectivement, tu ne commenceras pas, si tu as cinq groupes et, exemple, cinq dossiers par groupe, à regarder tous les dossiers. Je ne crois pas que tu vas prendre le temps dans un stage d'aller faire le tour de tous les dossiers. Par contre, si on se dresse un profil du groupe en demandant des situations problématiques, on pourra choisir d'aller consulter certains dossiers, surtout si l'enseignant nous dit que tel élève sera un défi dans le stage (G4;E55).

Un autre exemple concerne un participant qui s'exprimait à propos des rôles des intervenants et des savoirs collaboratifs : "Si tu as trouvé une stratégie qui fonctionne avec un élève, il faut la partager à son enseignant-tuteur, au directeur, afin de, peut-être, la mettre dans le plan d'intervention pour qu'on puisse partager la stratégie efficace » (G6; E95).

\section{Cinquième cas}

Enfin, le cinquième cas met en lumière une discussion autour des rôles des intervenants dans la gestion des troubles de comportement. Pendant son deuxième stage, une étudiante au BEPEP doit gérer une situation qui s'est déroulée sur la cour de récréation entre deux élèves, dont un présentant des troubles de comportement. Alors que l'enseignante est absente, la direction lui demande d'appeler les parents d'un des élèves, lequel a été légèrement blessé, afin de rapporter l'incident et d'intervenir auprès des deux élèves concernés. La stagiaire répond à la demande, mais elle se questionne à savoir si l'enseignante remplaçante ou la technicienne en éducation spécialisée de la classe n'auraient pas été mieux placées qu'elle pour le faire étant donné son statut de stagiaire. Elle demande l'avis du groupe par rapport à sa décision d'agir et d'appeler la mère de l'élève en question.

Les éléments qui ressortent de l'analyse des données autour de ce cas sont liés aux défis posés par la gestion de classe, avec 55 codes 


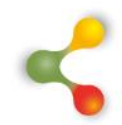

\section{REVUE HYBRIDE DE L'ÉDUCATION}

relevés en ce sens, et aux rôles des intervenants (39 codes). Du côté des savoirs professionnels, les savoirs pédagogiques ( 43 codes) et les savoirs collaboratifs (34 codes) s'avèrent les plus fréquemment relevés. Un participant énonce des propos liés à la gestion de classe et aux savoirs pédagogiques dans ces mots :

Un système d'émulation avec des couleurs, c'est toujours lié à des règles. Si lancer une motte de glace n'est pas dans tes règles, ça ne doit pas avoir d'impact sur ta couleur. Le système d'émulation-classe va avec les règles de la classe. Sur la cour, il y a des règles d'école. C'est important d'avoir des règles de vie claires que l'enfant comprend bien. Je ne dis pas que ce n'est pas un bon système les couleurs vert-jaunerouge. Je dis qu'il doit être bien structuré et compris par l'élève (G5;E97).

Un autre participant rapporte des propos associés aux rôles des intervenants et aux savoirs collaboratifs :

\begin{abstract}
Je pense que c'était à toi de téléphoner à la mère parce que tu étais la personne la plus signifiante à ce moment-là, surtout que l'enseignante et la TES n'étaient pas là. La première personne qui est responsable des comportements de son élève, c'est l'enseignante, et pas la direction. Le premier contact, c'est donc toi qui devais l'avoir. Pourquoi appeler le parent? Je pense que c'est pour assurer la collaboration avec la famille. Si c'est un enfant différent, il faut s'allier les parents et les mettre au courant de la situation pour qu'ils puissent continuer l'intervention à la maison (G5;E54).
\end{abstract}

\section{Discussion}

L'analyse des données a été réalisée sous l'angle des défis et des types de savoirs propres au développement des compétences professionnelles des stagiaires en enseignement.

Sans surprise, comme le résume le tableau 1, on constate que les thématiques abordées dans les groupes de codéveloppement professionnel sont très semblables aux défis rencontrés par les nouveaux enseignants et énoncés dans la problématique. La gestion de classe, la compréhension des rôles et des responsabilités des acteurs scolaires et universitaires, l'acte d'enseigner et les élèves ayant des besoins particuliers ont été relevés. L'analyse a fait émerger une autre catégorie, celle du contexte de formation inhérent aux stages en enseignement. 


\section{REVUE HYBRIDE DE L’ÉDUCATION}

Tableau 1: Les défis les plus fréquemment soulevés par les participants pendant les groupes de codéveloppement professionnel

\begin{tabular}{|c|c|c|c|c|c|}
\cline { 2 - 6 } \multicolumn{1}{c|}{} & \multicolumn{5}{c|}{ Principaux défis soulevés } \\
\hline Cas & $\begin{array}{c}\text { Gestion } \\
\text { de } \\
\text { classe }\end{array}$ & $\begin{array}{c}\text { Rôles et } \\
\text { responsabilités } \\
\text { des intervenants }\end{array}$ & $\begin{array}{c}\text { Contexte de } \\
\text { formation } \\
\text { (émergent) }\end{array}$ & $\begin{array}{c}\text { Acte } \\
\text { d'enseigner }\end{array}$ & $\begin{array}{c}\text { Élèves à } \\
\text { besoins } \\
\text { particuliers }\end{array}$ \\
\hline 1 & 96 & 44 & 38 & 37 & \\
\hline 2 & & 44 & & 31 & 53 \\
\hline 3 & & 39 & & & 58 \\
\hline 4 & & & & & \\
\hline 5 & 55 & & & & \\
\hline
\end{tabular}

Lors de la recension sur les catégories de savoirs professionnels, l'interprétation initiale faite du défi lié aux rôles et responsabilités des intervenants se rapportait à l'exercice de la profession en milieu scolaire ; elle ne concernait pas le stagiaire en formation. Ainsi, la catégorie émergente du contexte de formation a pris forme. À la lumière de l'analyse, il appert que le contexte de formation se révèle du même ordre que celui des rôles et responsabilités, ce que montre la ligne pointillée dans le tableau. Nous proposons donc de ne retenir que le premier et d'élargir son interprétation pour inclure le positionnement, non seulement de l'enseignant par rapport aux autres acteurs, mais également celui du stagiaire en formation. Ce dernier voit effectivement son rôle et ses responsabilités évoluer, et ce, par rapport aux autres intervenants, au fil des stages et de son insertion dans la profession.

De son côté, le tableau 2 rappelle les principaux savoirs professionnels partagés entre les participants des groupes de codéveloppement. Ces derniers demeurent aussi cohérents avec les objets de discussion. 
REVUE HYBRIDE DE L'ÉDUCATION

Tableau 2: Les savoirs professionnels les plus fréquemment évoqués par les participants lors des groupes de codéveloppement professionnel.

\begin{tabular}{|c|c|c|c|c|c|}
\cline { 2 - 6 } \multicolumn{1}{c|}{} & \multicolumn{5}{c|}{ Principaux savoirs professionnels évoqués } \\
\hline Cas & Pédagogiques & Expérientiels & $\begin{array}{c}\text { Collaboratifs } \\
\text { (émergents) }\end{array}$ & $\begin{array}{c}\text { Identitaires } \\
\text { (émergents) }\end{array}$ & $\begin{array}{c}\text { Sur les } \\
\text { élèves }\end{array}$ \\
\hline 1 & 62 & 48 & & & \\
\hline 2 & & & 30 & 29 & \\
\hline 3 & 29 & & & & 31 \\
\hline 4 & 32 & & 39 & & \\
\hline 5 & 43 & & 34 & & \\
\hline
\end{tabular}

Cette mise en lumière de la somme des codes attribués autour des savoirs partagés lors des groupes de codéveloppement montre des discussions fortement imprégnées de savoirs pédagogiques, c'est-à-dire des connaissances portant sur la gestion et l'organisation de la classe, les méthodes et les stratégies pédagogiques à mettre en place pour faire apprendre et adopter des règles de fonctionnement en classe. Ce tableau présente également le nombre de codes liés aux savoirs partagés sur les élèves, c'est-à-dire des connaissances, des caractéristiques et des besoins des élèves.

II expose enfin des savoirs émergents, c'est-à-dire des savoirs collaboratifs et identitaires. Les savoirs collaboratifs s'associent à la relation de collaboration qui doit primer avec les collègues, les intervenants et les parents afin de favoriser la réussite des élèves, et ce, au regard des difficultés que comporte cette entreprise. Quant aux savoirs identitaires, ils se rapportent aux rôles et aux responsabilités des stagiaires et des différents acteurs scolaires et universitaires, ainsi qu'au positionnement de soi en tant que professionnels, éthique et responsable, au sein de ce groupe professionnel. Dans cette perspective, Vanhulle (2013) rapportait que, construire ses savoirs professionnels, c'est se construire soi-même en tant que professionnel. Ce serait donc normal de voir émerger autant les savoirs identitaires et collaboratifs ; les premiers permettant de se définir professionnellement et les seconds favorisant l'émergence de lien avec d'autres intervenants.

Par ailleurs, l'analyse des données et les tableaux ne montrent pas ou peu de savoirs de référence, qu'ils soient théoriques, disciplinaires ou 


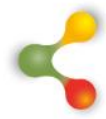

\section{REVUE HYBRIDE DE L'ÉDUCATION}

académiques. En fait, à l'instar de Breithaupt (2015), les savoirs académiques ne sont pas ou peu définis dans le langage quotidien employé par les stagiaires, et par conséquent, ne sont pas différenciés des savoirs de la pratique. Le peu de références au langage académique pourrait s'expliquer par la situation de communication qui demande d'adapter le langage utilisé en fonction des interlocuteurs.

\section{Conclusion}

Cet article rapporte les résultats d'une recherche-action-formation menée avec des stagiaires de trois modules en enseignement, ainsi que des intervenants de plusieurs commissions scolaires. II met en évidence la prépondérance de certains défis qui préoccupent les stagiaires en enseignement au-delà de leurs modules respectifs. Ainsi, les codes relevés à l'égard des défis relatifs à la gestion de classe, aux élèves ayant des besoins particuliers et à l'acte d'enseigner coïncident avec ceux associés à l'insertion dans la profession. Toutefois, en combinant les segments portant sur les difficultés liées aux rôles et responsabilités des intervenants et ceux rattachés au contexte de formation, ce défi apparait comme prioritaire. Le fait de devoir se définir en tant que futur enseignant, et ce, par rapport aux autres intervenants, semble constituer le principal enjeu lors de la formation initiale.

De même, l'article relève deux nouvelles catégories de savoirs professionnels partagés par les stagiaires en enseignement ; les savoirs identitaires et collaboratifs. Ces derniers soulignent le besoin de construire des savoirs pour faire face au défi le plus important, celui de définir son rôle et ses responsabilités au fil des stages. Ces savoirs, comme ce défi, rappellent l'importante place qu'occupe la compétence portant sur l'identité professionnelle, pendant les stages. À ce titre, se pourrait-il que les différents défis et savoirs relevés recoupent les différentes compétences professionnelles ? Ce langage qui réunit les milieux universitaire et scolaire du Québec pourrait-il servir de point de référence pour échanger autour des défis à relever et des savoirs à développer?

Ces questions nous ramènent à l'enjeu de la collaboration entre les milieux et les acteurs qui les composent. À la fin de la première année, les participants à la recherche ont suggéré des pistes pour favoriser cette collaboration, dont, notamment, miser sur l'interprofessionnalité et l'intermodularité en intégrant le groupe de codéveloppement professionnel aux cursus universitaires. Ces premières propositions seront bonifiées par le travail réalisé lors de la deuxième année de l'étude, laquelle sera entièrement consacrée à l'identification de stratégies collaboratives université-milieu scolaire. 


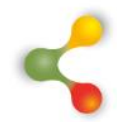

\section{REVUE HYBRIDE DE L'ÉDUCATION}

\section{Références}

Balslev, K. (2016). Soutenir la construction de savoirs professionnels des futurs enseignants dans les entretiens de stage. Didactiques en pratique, $1(2), 12-20$.

Beaumont, C., Lavoie, J. et Couture, C. (2010). Les pratiques collaboratives en milieu scolaire: cadre de référence pour soutenir la formation. Centre de recherche et d'intervention sur la réussite scolaire.

Borges, C., Portelance, L. et Pharand, J. (2011). La collaboration entre les agents éducatifs: de la prescription aux pratiques. Dans L. Portelance, C. Borges et J. Pharand (dir.), La collaboration dans le milieu de l'éducation: dimension pratiques et perspectives théoriques (p. 2-9). Presses de l'Université du Québec.

Breithaupt, S. (2015). Nature et usage des savoirs de référence lors des visites de stage. e-JIREF, 1(3), 43-56.

Bush, G. (2003). The School Buddy System: The Practice of Collaboration. American Library Association.

Clerc, A. J. (2013). Rôle des savoirs théoriques de référence dans les parcours de formation des futurs enseignants des premiers degrés de la scolarité [thèse de doctorat, Université de Genève]. https://archive-ouverte.unige.ch/unige:28992

Clot, Y., Faïta, D., Fernandez, G. et Scheller, L. (2001). Les entretiens en autoconfrontation croisée : une méthode en clinique de l'activité. Education Permanente, 1(146), 17-25.

Cody, N., Coulombe, S., Giroux, P., Gauthier, D. et Gaudreault., S. (2016). Pratiques, objets et finalités de collaboration en lien avec l'intégration des tablettes numériques dans une école secondaire. Canadian Journal of Learning and Technologies, 42(3), 1-16.

Cody, N. (2013). La formation d'un enseignant au préscolaire-primaire. Une interaction constante entre les activités théoriques, pratiques et réflexives. Presses académiques francophones.

Collin, S. (2010). L'interaction en ligne comme soutien à la pratique réflexive des enseignants-stagiaires [thèse de doctorat, Université de Montréal].

Papyrus. https://papyrus.bib.umontreal.ca/xmlui/handle/1866/4546

Colognesi, S. et Van Nieuwenhoven, C. (2017). Le processus de coévaluation entre superviseurs et étudiants en formation initiale des enseignants du primaire. Canadian Journal of Education/Revue canadienne de l'éducation, 40(2), 1-27. 


\section{8}

\section{REVUE HYBRIDE DE L'ÉDUCATION}

Corriveau, L. et Savoie Zajc, L. (2010). Introduction. Dans L. Corriveau, C. Letor et D. Périsset Bagnoud (dir.), Travailler ensemble dans les établissements scolaires et de formation: processus, stratégies, paradoxes (p. 7-12). De Boeck Supérieurs.

Coulombe, S., Allaire, S. et Zourhlal, A. (2016). Une communauté de pratique en ligne pour soutenir les nouveaux enseignants en formation professionnelle. Dans C. Gagnon et S. Coulombe (dir.), Enjeux et défis de la formation à l'enseignement professionnel (p. 99-116). Presses de l'Université du Québec.

Coulombe, S., Doucet, M., Zourhlal, A., et Thibeault, S. (2017). Collaborer pour soutenir les nouveaux enseignants en formation professionnelle, enjeu d'une recherche-action. Revue hybride de l'éducation, 1(1), 152-172.

Coulombe, S., Zourhlal, A. et Allaire, S. (2010). Des obstacles à l'insertion des nouveaux enseignants en formation professionnelle. Formation et Profession, 17(2), 25-28.

De Stercke, J., De Lièvre, B., Temperman, G., Cambier, J. B., Renson, J. M., Beckers, J., Leemans, M. et Marechal, C. (2010). Difficultés d'insertion professionnelle dans l'enseignement secondaire ordinaire en Belgique francophone. La formation des enseignants, e-294, 136-148.

Desbiens, J.-F., Spallanzani, C. et Borges, C. (2013). Quand le stage en enseignement déraille. Regards pluriels sur une réalité trop souvent occultée. Presses de l'Université du Québec.

Dionne, L. et Savoie Zajc, L. (2011). Sens, caractéristiques et retombées de la collaboration entre enseignants et contribution au développement professionnel. Dans L. Portelance, C. Borges et J. Pharand (dir.), La collaboration dans le milieu de l'éducation: dimensions pratiques et perspectives théoriques (p. 45-60). Presses de l'Université du Québec.

Duchesne, C. et Kane, R. (2010). Défis de l'insertion professionnelle et dispositifs d'encadrement. McGill Journal of Education/Revue des sciences de l'éducation de McGill, 45(1), 63-80.

Fenstermacher, G. et Richardson, V. (1994). L'explicitation et la reconstruction des arguments pratiques dans l'enseignement. Cahiers de la recherche en éducation, 1(1), 157-182.

Gagné, A. (2019). L'apport de l'expérience professionnelle à la construction de l'identité d'enseignant associé en enseignement professionnel [thèse de doctorat, Université du Québec à Chicoutimi]. Constellation.

https://constellation.uqac.ca/5270/1/GagnxE9 uqac 0862D 1059 4.pdf 


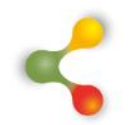

\section{REVUE HYBRIDE DE L'ÉDUCATION}

Garnier, B. (2008). Circulation des savoirs entre universitaires et pédagogues dans une situation d'accompagnement. Recherche et formation, 1(57), 115-128.

Koehler, M. et Mishra, P. (2009). What is technological pedagogical content knowledge (TPACK)?. Contemporary issues in technology and teacher education, 9(1), 60-70.

Larivée, S., Kalubi, J. C. et Terrisse, B. (2006). La collaboration écolefamille en contexte d'inclusion: entre obstacles, risques et facteurs de réussite. Revue des sciences de l'éducation, 32(3), 525-543.

Lessard, C., Kamanzi, P. C. et Larochelle, M. (2009). De quelques facteurs facilitant l'intensification de la collaboration au travail parmi les enseignants: le cas des enseignants canadiens. Éducation et sociétés, (1), 59-77.

L'Hostie, M., Cody, N., et Laurin, N. (2011). Caractéristiques et particularités du groupe de discussion favorisé dans un dispositif de recherche collaborative en éducation. Recherches qualitatives, 29(3), 198-213.

Malo, A. (1997). Exploration de la notion de transformation à partir d'une analyse critique du concept de connaissance pédagogique de la matière de L.S. Shulman [mémoire de maîtrise inédit, Université Laval].

Malo, A. (2005). Parcours évolutif d'un savoir professionnel : une étude de cas multiples menée auprès de futurs enseignantes et enseignants du secondaire en stage intensif [thèse de doctorat, Université Laval] http://www.crifpe.ca/publications/view/3328/p 3

Marcel, J.-F., Dupriez, V. et Bagnoud, D. P. (2007). Le métier d'enseignant : nouvelles pratiques, nouvelles recherches. Dans J.-F. Marcel, V. Dupriez, D. P. Bagnoud et M. Tardif (dir.), Coordonner, collaborer, coopérer : de nouvelles pratiques enseignantes (p. 7-17). De Boeck Université.

Martin, D. (2002). Terrain et théories dans les réformes de la formation des maîtres. Dans M. Carbonneau et M. Tardif (dir.), Les réformes en éducation, leurs impacts sur l'école (p. 95-109). Éditions du CRP.

Mialaret, G. et Barbier, J.M. (2011). Savoirs théoriques, savoirs scientifiques et savoirs d'action en éducation. Dans J-M. Barbier (dir.), Savoirs théoriques et savoirs d'action (p. 161-187). Presses Universitaires de France.

Morales Perleza, A. (2016). Les savoirs professionnels à la base de la formation des enseignants au Québec et en Ontario : une étude comparative des modèles universitaires de professionnalisation et de leurs enjeux [thèse de doctorat, Université de Montréal]. Papyrus. https://papyrus.bib.umontreal.ca/xmlui/handle/1866/18598 


\section{$\&$}

\section{REVUE HYBRIDE DE L'ÉDUCATION}

Mukamurera, J., Martineau, S., Bouthiette, M. et Ndoreraho, J. P. (2013). Les programmes d'insertion professionnelle des enseignants dans les commissions scolaires du Québec : portrait et appréciation des acteurs. Éducation et formation, 299(1), 13-35.

Paillé, P. (1994). Pour une méthodologie de la complexité en éducation : le cas d'une recherche-action-formation. Revue canadienne de l'éducation, 19(3), 215-230.

Paillé, P. et Mucchielli, A. (2008). L'analyse qualitative en sciences humaines et sociales. ( $2^{\mathrm{e}}$ éd.). Armand Colin.

Pastré, P., Mayen, P. et Vergnaud, G. (2006). La didactique professionnelle. Revue française de pédagogie. Recherches en éducation, 1(154), 145-198.

Payette, A., et Champagne, C. (1997). Le groupe de codéveloppement professionnel. Presses de l'Université du Québec.

Perez-Roux, T. (2016). Transitions professionnelles et transactions identitaires ; expériences, épreuves, ouvertures. Pensée plurielle, 41(1), 81-93.

Perrenoud, P. (2001). L'analyse collective des pratiques pédagogiques peut-elle transformer les praticiens. Dans P. Perrenoud (dir.), Développer la pratique réflexive dans le métier d'enseignant: professionnalisation et raison pédagogique (p. 17-34). ESF.

Perrenoud, P., Altet, M. et Lessard, C. (2008). Conflits de savoirs en formation des enseignants : entre savoirs issus de la recherche et savoirs issus de l'expérience. De Boeck Supérieur.

Prud'homme, L. (2007). La différenciation pédagogique : analyse du sens construit par des enseignantes et un chercheur-formateur dans un contexte de recherche-action-formation [thèse de doctorat inédite, Université du Québec à Montréal].

Prud'Homme, L., Presseau, A. et Dolbec, A. (2007). Une pratique réflexive de recherches «à trait d'union»: quels enjeux scientifiques? Recherches qualitatives, 4(hors-série), 36-68.

Rojo, S. et Minier, P. (2015). Les facteurs de stress reconnus comme sources de l'abandon de la profession enseignante au secondaire au Québec. Éducation et francophonie, 43(2), 219-240.

Schon, D. A. (1994). Le praticien réflexif : à la recherche du savoir caché dans l'agir professionnel. Logiques.

Tardif, M. et Lessard, C. (1999). Le travail enseignant : contribution à l'étude du travail dans les métiers et les professions d'interactions humaines. Presses de l'Université Laval. 


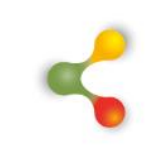

\section{REVUE HYBRIDE DE L'ÉDUCATION}

Theureau, J. (2000). Anthropologie cognitive et analyse des compétences. Dans J-M., Barbier (dir.), L'analyse de la singularité de l'action (p. 171-211). Presses Universitaires de France.

Vanhulle, S. (2009). Savoirs professionnels et construction sociodiscursive de l'agir. Bulletin VALS-ASLA, 90(1), 167-188.

Vanhulle, S. (2012). Quand l'activité professionnelle s'invite dans la formation académique: le cas de stages en enseignement. Dans $\mathrm{E}$. Bourgeois et M. Durand (dir.). Apprendre au travail (p.165-175). Presses universitaires de France.

Vanhulle, S. (2013). Dialoguer avec le discours des professionnels en devenir. Ikastaria. Cuadernos de educación, 1(19), 37-67.

Verloop, N., Van Driel, J. et Meijer, P. (2001). Teacher knowledge and the knowledge base of teaching. International Journal of Educational Research, 35(1), 441-461.

Vermersch, P. (2004). Aide à l'explicitation et retour réflexif. Éducation permanente, 1(160), 71-80. 